\title{
Geometric Disturbance Decoupling Control of Vehicles with Active Suspensions
}

\author{
D. Prattichizzo ${ }^{\dagger}$, P. Mercorelli ${ }^{\dagger}$, A. Bicchi ${ }^{\ddagger}$ A. Vicino ${ }^{\dagger}$ \\ ${ }^{\dagger}$ Dipartimento di Ingegneria dell'Informazione, Università di Siena, via Roma 56, 53100 Siena, Italia \\ prattichizzo[mercorel] \{vicino\}@ing.unisi.it \\ ‡ Centro "E. Piaggio", Università di Pisa, via Diotisalvi 2, 56100 Pisa, Italia \\ bicchi@piaggio.ccii.unipi.it
}

\begin{abstract}
In this paper the problem of localizing disturbances of a vehicle with active suspensions is investigated. We focus on the regulation of the chassis posture in spite of unaccessible disturbances. The regulated variables, i.e. the roll and pitch angles and the chassis heights, can be disturbance decoupled by state feedback. The decoupling problem is analyzed from a geometric control point of view. The geometric aspects of the vehicle dynamics are emphasized and it is shown that disturbance localization is a structural property of vehicles with active suspensions. On the implementation side, we show that, if suspensions heights along with their derivatives are available as measured outputs, disturbance localization can be gained by a static output feedback.
\end{abstract}

\section{Introduction}

The actuation of suspensions along with proper sensor systems allows the vehicle controller to actively reject external disturbances to enhance both ride comfort and safety. In most of the conventional cars, rejection of disturbances is gained by passive devices which have a damping force fixed at all frequencies and cannot attenuate both low and high frequency vibrations. On the contrary, active suspensions are able to change the damping force according to the sensed vibrations and can improve performances of the whole system.

The control of active suspensions has been widely investigated in literature. In [3] Hrovat studied the problem of optimal design of active suspensions by casting it into an equivalent linear-quadratic (LQG)optimization problem.

Two different types of disturbances can influence vehicle dynamics. One acts directly on the sprung mass of the vehicle and for instance can be generated by lateral accelerations, the other type of disturbances is due to road irregularity and is transmitted through the same suspensions. In this paper we focus on the last type of disturbances and our purpose is to isolate the chassis from vibrations transmitted through suspensions. The paper is aimed at the synthesis of a decoupling control law making the regulated outputs, i.e. roll, pitch and chassis height, insensitive to the external disturbances. A geometric approach to the control problem is adopted. The earliest geometric approach to noninteracting and decoupling control are due to Basile and Marro [1, 2] and to Wonham and Morse [7].

\section{Dynamic model of the vehicle}

The mathematical model of vertical dynamics of road vehicles is derived. The mechanical structure of the vehicle is reported in figure 1 . The vehicle consists of a rigid chassis and two rigid front and rear axes. The sprung mass is linked with these axes by means of four passive suspensions and actuators. An independent control action is exerted at each corner of the vehicle. The controlled vertical force $u_{j}(j=1, \ldots, 4)$ is generated at the expense of additional energy source such as compressors or pumps.

Assume that the vehicle is in an equilibrium configuration (see Figure 1 for illustration) and that roll center coincides with gravity center. Let us introduce some notation.

$\theta_{r}$ : variation of the roll angle around the equilibrium configuration;

$I_{r}$ : moment of inertia of the chassis about the roll axis; $\theta_{p}$ : variation of the pitch angle around the equilibrium configuration;

$I_{p}$ : moment of inertia of the chassis about the pitch axis;

$z$ : variation of the height of the center of gravity (CG) of the sprung mass;

$M_{b}$ : sprung mass;

$\theta_{a i}$ : variation angles of the front $(i=1)$ and of the rear axis $(i=2)$;

\section{0-7803-4104-X/98/\$10.00 @1998 IEEE}



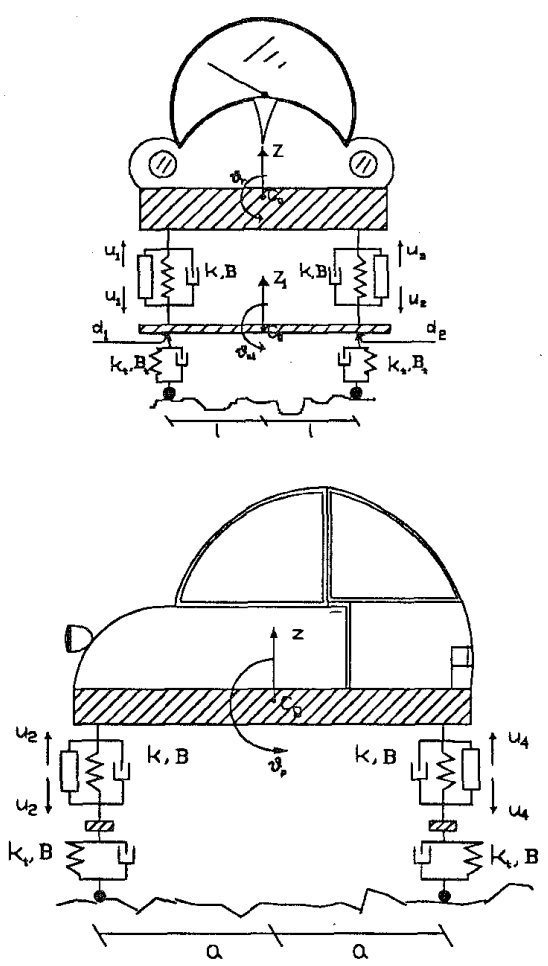

Figure 1: Mechanical model of a vehicle with active suspensions. Front and side view.

$I_{a i}$ : moment of inertia of the $i$-th axis; $z_{i}$ : variation of the height of the CG of the $i$-th axis; $M_{a i}$ : mass of the $i$-th axis;

$k, \beta$ : spring and damping coefficient of the passive part of suspensions;

$k_{t}, \beta_{t}$ : visco-elastic parameters of tires;

$a$ : half distance between front (or rear) suspensions;

$l$ : half distance between of the two axes;

$d_{j}$ : independent, unaccessible, external disturbances exerted on the axes;

The adopted model has 7 degrees-of freedom and takes into account the roll, the pitch angles of the chassis, the rotations of wheel axes and the vertical displacements of the sprung mass and of the two axes. Lateral and longitudinal dynamics of the sprung mass are not considered in this model.

Equality of visco-elastic parameters of the passive suspensions has been assumed. Notice that such an assumption can be easily satisfied by means of a proper compensating control for the vertical forces $u_{i}^{\prime}$ 's. The consequence of this assumption is that dynamics of pitch, roll and vertical motions are decoupled and can be independently analyzed.

Under the assumption that $\theta_{r}, \theta_{p}, z, \theta_{a 1}, \theta_{a 2}, z_{1}$ and $z_{2}$ are small, linear approximation of system dynamics are simply obtained as follows.

Chassis dynamics $\left(\theta_{r}, \theta_{p}, z\right)$.

$$
\begin{gathered}
I_{r} \ddot{\theta}_{r}=-4 k l^{2} \theta_{r}-4 \beta l^{2} \dot{\theta}_{r}+2 k l^{2} \theta_{a 1}+2 \beta l^{2} \dot{\theta}_{a 1}+ \\
2 k l^{2} \theta_{a 2}+2 \beta l^{2} \dot{\theta}_{a 2}+\left(u_{2}-u_{1}\right) l+\left(u_{4}-u_{3}\right) l \\
I_{p} \ddot{\theta}_{p}=-4 k a^{2} \theta_{p}-4 \beta a^{2} \dot{\theta}_{p}-2 k z_{1} a-2 \beta \dot{z}_{1} a+ \\
2 k z_{2} a+2 \beta \dot{z}_{2} a+\left(u_{3}-u_{1}\right) a+\left(u_{4}-u_{2}\right) a \\
M_{b} \ddot{z}=-4 k z-4 \beta \dot{z}+2 k z_{1}+2 \beta \dot{z}_{1}+ \\
2 k z_{2}+2 \beta \dot{z}_{2}+\left(u_{1}+u_{2}+u_{3}+u_{4}\right)
\end{gathered}
$$

Axes dynamics $\left(\theta_{a i}, z_{i}\right)$.

$$
\begin{aligned}
& I_{a 1} \ddot{\theta}_{a 1}=-2\left(k_{t}+k\right) l^{2} \theta_{a 1}-2\left(\beta_{t}+\beta\right) l^{2} \dot{\theta}_{a 1}+2 k l^{2} \theta_{r}+ \\
& 2 \beta l^{2} \dot{\theta}_{r}-\left(u_{2}-u_{1}\right) l-d_{1} l+d_{2} l ; \\
& I_{a 2} \ddot{\theta}_{a 2}=-2\left(k_{t}+k\right) l^{2} \theta_{a 2}-2\left(\beta_{t}+\beta\right) l^{2} \dot{\theta}_{a 2}+2 k l^{2} \theta_{r}+ \\
& \quad 2 \beta l^{2} \dot{\theta}_{r}-\left(u_{4}-u_{3}\right) l-d_{3} l+d_{4} l ; \\
& M_{a 1} \ddot{z}_{1}=-2\left(k_{t}+k\right) z_{1}-2\left(\beta_{t}+\beta\right) \dot{z}_{1}-2 k a \theta_{p}-2 \beta a \dot{\theta}_{p}+ \\
& 2 k z+2 \beta \dot{z}+d_{1}+d_{2}-\left(u_{1}+u_{2}\right) ; \\
& M_{a 2} \ddot{z}_{2}=-2\left(k_{t}+k\right) z_{2}-2\left(\beta_{t}+\beta\right) \dot{z}_{2}+2 k a \theta_{p}+2 \beta a \dot{\theta}_{p}+ \\
& 2 k z+2 \beta \dot{z}+d_{3}+d_{4}-\left(u_{3}+u_{4}\right) .
\end{aligned}
$$

Sign conventions for forces, motion and other parameters of vehicle dynamics are defined in Figure 1.

\subsection{State space model}

In this paper we are interested in controlling the chassis posture in spite of disturbances $d_{j}$ transmitted through the suspensions and generated by road irregularities. Such a type of regulation will be referred to as ride heights regulation [6] and consists in controlling the roll and pitch angles and the height of the sprung mass CG. Thus the output vector is defined as

$$
\mathbf{y}=\left(\theta_{r}, \theta_{p}, z\right)^{T}
$$

In the following the vehicle dynamics is described in the state space domain.

Let us define the 14-dimensional state vector $\mathrm{x}$, the 4-dimensional input vector and the 4-dimensional disturbance vector as

$$
\begin{aligned}
& \mathrm{x}=\left(\mathrm{x}_{\mathrm{r}}^{\mathbf{T}} \mathrm{x}_{\mathrm{v}}^{\mathbf{T}}\right)^{\mathbf{T}} ; \quad \text { where } \\
& \mathrm{x}_{\mathbf{r}}=\left(\begin{array}{llllll}
\theta_{\mathbf{r}} & \theta_{\mathrm{a} 1} & \theta_{\mathrm{a} 2} & \dot{\theta}_{\mathbf{r}} & \dot{\theta}_{\mathrm{a} 1} & \dot{\theta}_{\mathrm{a} 2}
\end{array}\right)^{\mathbf{T}} ;
\end{aligned}
$$

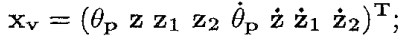

$$
\begin{aligned}
& \mathbf{u}=\left(\begin{array}{llll}
\mathbf{u}_{1} & \mathbf{u}_{2} & \mathbf{u}_{3} & \mathbf{u}_{4}
\end{array}\right) ; \\
& d=\left(\begin{array}{llll}
d_{1} & d_{2} & d_{3} & d_{4}
\end{array}\right) .
\end{aligned}
$$


Notice that we have grouped the roll dynamics in vector $\mathrm{x}_{\mathbf{r}}$, while the vector $\mathrm{x}_{\mathbf{v}}$ contains the pitch and the vertical dynamics.

From the chassis and axes dynamics the state space model of linearized dynamics around the equilibrium configuration is simply obtained as

$$
\left\{\begin{array}{l}
\dot{\mathrm{x}}=\mathrm{Ax}+\mathrm{Bu}+\mathrm{Dd} \\
\mathrm{y}=\mathrm{Cx}
\end{array}\right.
$$

where the state matrix is

$$
\mathbf{A}=\left[\begin{array}{cc}
\mathbf{A}_{11} & \mathbf{0}_{(6 \times 8)} \\
\mathbf{0}_{(8 \times 6)} & \mathbf{A}_{22}
\end{array}\right]
$$

with

$$
\begin{aligned}
& \mathbf{A}_{11}=\left[\begin{array}{cc}
\mathbf{0}_{3} & \mathbf{I}_{3} \\
\mathbf{M}_{1 \mathrm{k}} & \mathbf{M}_{1 \beta}
\end{array}\right] ; \quad \mathbf{A}_{22}=\left[\begin{array}{cc}
\mathbf{0}_{4} & \mathbf{I}_{4} \\
\mathbf{M}_{2 \mathrm{k}} & \mathbf{M}_{2 \beta}
\end{array}\right] \\
& \mathbf{M}_{1 k}=\left[\begin{array}{ccc}
\frac{-4 k l^{2}}{I_{r}} & \frac{2 k l^{2}}{I_{r}} & \frac{2 k l^{2}}{I_{r}} \\
\frac{2 k l^{2}}{I_{a 1}} & \frac{-2\left(k_{t}+k\right) l^{2}}{I_{a 1}} & 0 \\
\frac{2 k l^{2}}{I_{a 2}} & 0 & \frac{-2\left(k_{t}+k\right) l^{2}}{I_{a 2}}
\end{array}\right] \text {; } \\
& \mathbf{M}_{1 \beta}=\left[\begin{array}{ccc}
\frac{-4 \beta l^{2}}{I_{r}} & \frac{2 \beta l^{2}}{I_{r}} & \frac{2 \beta l^{2}}{I_{r}} \\
\frac{2 \beta l^{2}}{I_{a 1}} & \frac{-2\left(\beta_{t}+\beta\right) l^{2}}{I_{a 1}} & 0 \\
\frac{2 \beta l^{2}}{I_{a 2}} & 0 & \frac{-2\left(\beta_{t}+\beta\right) l^{2}}{I_{a 2}}
\end{array}\right] \text {; } \\
& \mathbf{M}_{2 k}=\left[\begin{array}{cccc}
\frac{-4 k a^{2}}{I_{p}} & 0 & \frac{-2 k a}{I_{p}} & \frac{2 k a}{I_{p}} \\
0 & \frac{-4 k}{M_{b}} & \frac{2 k}{M_{b}} & \frac{2 k}{M_{b}} \\
-\frac{2 k a}{M_{a 1}} & \frac{2 k}{M_{a 1}} & \frac{-2\left(k_{t}+k\right)}{M_{a 1}} & 0 \\
\frac{2 k a}{M_{a 2}} & \frac{2 k}{M_{a 2}} & 0 & \frac{-2\left(k_{t}+k\right)}{M_{a 2}}
\end{array}\right] \text {; } \\
& \mathbf{M}_{2 \beta}=\left[\begin{array}{cccc}
\frac{-4 \beta a^{2}}{I_{p}} & 0 & \frac{-2 \beta a}{I_{p}} & \frac{2 \beta a}{I_{p}} \\
0 & \frac{-4 \beta}{M_{b}} & \frac{2 \beta}{M_{b}} & \frac{2 \beta}{M_{b}} \\
-\frac{2 \beta a}{M_{a 1}} & \frac{2 \beta}{M_{a 1}} & \frac{-2\left(\beta_{t}+\beta\right)}{M_{a 1}} & 0 \\
\frac{2 \beta a}{M_{a 2}} & \frac{2 \beta}{M_{a 2}} & 0 & \frac{-2\left(\beta_{t}+\beta\right)}{M_{a 2}}
\end{array}\right] \text {, }
\end{aligned}
$$

the input matrix is

$$
\mathbf{B}=\left[\begin{array}{l}
\mathbf{B}_{1} \\
\mathbf{B}_{2}
\end{array}\right]
$$

with

$$
\begin{aligned}
& \mathbf{B}_{1}=\left[\begin{array}{c}
0_{(3 \times 4)} \\
\mathbf{B}_{1 \mathrm{~L}}
\end{array}\right] ; \quad \mathbf{B}_{2}=\left[\begin{array}{c}
\mathbf{0}_{4} \\
\mathbf{B}_{2 \mathrm{~L}}
\end{array}\right] ; \\
& \mathbf{B}_{1 \mathrm{~L}}=\left[\begin{array}{cccc}
\frac{-l}{I_{f}} & \frac{l}{I_{r}} & \frac{-l}{I_{r}} & \frac{l}{I_{r}} \\
\frac{l}{I_{a 1}} & -\frac{l}{I_{a 1}} & 0 & 0 \\
0 & 0 & \frac{l}{I_{a 2}} & -\frac{l}{I_{\mathrm{a} 2}}
\end{array}\right] ; \\
& \mathbf{B}_{2 L}=\left[\begin{array}{cccc}
\frac{-a}{I_{p}} & \frac{-a}{I_{p}} & \frac{a}{I_{p}} & \frac{a}{I_{p}} \\
\frac{l}{M_{b}} & \frac{l}{M_{b}} & \frac{l}{M_{b}} & \frac{l}{M_{b}} \\
\frac{-1}{M_{a 1}} & \frac{-1}{M_{a 1}} & 0 & 0 \\
0 & 0 & \frac{-1}{M_{\mathrm{a} 2}} & \frac{-1}{M_{a 2}}
\end{array}\right]
\end{aligned}
$$

the disturbance matrix is

$$
\mathbf{D}=\left[\begin{array}{l}
\mathbf{D}_{1} \\
\mathbf{D}_{2}
\end{array}\right]
$$

with

$$
\begin{gathered}
\mathbf{D}_{1}=\left[\begin{array}{c}
\mathbf{0}_{(3 \times 4)} \\
\mathbf{D}_{1 \mathbf{L}}
\end{array}\right] ; \quad \mathbf{D}_{2}=\left[\begin{array}{c}
\mathbf{0}_{4} \\
\mathbf{D}_{2 \mathbf{L}}
\end{array}\right] ; \\
\mathbf{D}_{1 L}=\left[\begin{array}{cccc}
0 & 0 & 0 & 0 \\
\frac{-l}{I_{a 1}} & \frac{l}{I_{a 1}} & 0 & 0 \\
0 & 0 & \frac{-l}{I_{a 2}} & \frac{l}{I_{a 2}}
\end{array}\right] ; \\
\mathbf{D}_{2 L}=\left[\begin{array}{cccc}
0 & 0 & 0 & 0 \\
0 & 0 & 0 & 0 \\
\frac{1}{M_{a 1}} & \frac{1}{M_{a 1}} & 0 & 0 \\
0 & 0 & \frac{1}{M_{a 2}} & \frac{1}{M_{a 2}}
\end{array}\right] ;
\end{gathered}
$$

and finally the output matrix is

$\mathrm{C}=\left[\begin{array}{c|c|c|c}1 & 0_{(3 \times 5)} & 0_{(1 \times 2)} & 0_{(3 \times 6)} \\ 0_{(2 \times 1)} & \mathbf{I}_{2} & \end{array}\right]$

\section{Localization of disturbances}

On the base of previous formulation of vehicle $\mathrm{dy}$ namics, the ride heights regulation can be rigorously stated as a problem of unaccessible disturbance localization. This problem consists in realizing a state feedback $\mathbf{u}=\mathbf{F x}$, such that, starting at zero state, the regulated output $\mathbf{y}(t)$ is identically zero for all the admissible disturbances $\mathbf{d}(\mathbf{t})$.

We attack the problem by using classical tools of the geometric control theory. It is well known that the unaccessible disturbance localization problem has a solution if and only if there exists a matrix $\mathbf{F}$ such that $\min \mathcal{I}(\mathbf{A}+\mathbf{B F}, \mathbf{D})$, the minimal $(\mathbf{A}+\mathbf{B F})$-invariant subspace containing the column space of the disturbance matrix $\mathbf{D}$, is included in the nullspace of the regulated output matrix, $\operatorname{ker}(\mathbf{C})$. Since this condition depends on the choice of $\mathbf{F}$, it lacks convenience and an equivalent structural condition is preferred [2].

Proposition 1 The unaccessible disturbance localization problem is solvable if and only if

$$
\operatorname{im}(\mathbf{D}) \subseteq \mathbf{V}^{*}
$$

where $\mathbf{V}^{*}=\max \mathcal{V}(\mathbf{A}, \mathbf{B}, \operatorname{ker}(\mathbf{C}))$ is the maximal (A, B)-controlled invariant contained in $\operatorname{ker}(\mathbf{C})$ and $\mathrm{im}(\mathbf{D})$ is the column space of the disturbance matrix.

For the localization problem to be technically sound, we should require that the state feedback, other than localizing disturbance in the nullspace of the output matrix, stabilizes the whole system in its equilibrium point. The following theorem shows that the unaccessible disturbance localization with stability for the regulated output $\mathbf{y}$ of the dynamic system in (5), is an intrinsic structural property of vehicle with active suspensions. 
Theorem 1 (Disturbance localization.) For the dynamic system (5) of a vehicle with active suspensions, there always exists a stabilizing state feedback gain $\mathbf{F}$ which localizes disturbances $\mathbf{d}$ in the nullspace of the regulated output $\mathbf{y}=\left(\theta_{r}, \theta_{p}, z\right)$.

Sketch of the proof. (For the complete proof the reader is referred to [5].) The proof starts with the definition of the column space of matrix $\mathbf{J}$,

$$
\mathbf{J}=\left[\begin{array}{cc}
\mathbf{J}_{1} & 0 \\
0 & \mathrm{~J}_{2}
\end{array}\right]
$$

where

$J_{1}=\left[\begin{array}{c|c}{ }^{0}(1 \times 2) & { }_{I_{2}}{ }_{(3 \times 2)} \\ \hline{ }^{0}(3 \times 2) & { }^{0}{ }_{(1 \times 2)}\end{array}\right] ; J_{2}=\left[\begin{array}{c|c}{ }^{0}(2 \times 2) & { }_{I_{2}}{ }_{(4 \times 2)} \\ \hline{ }^{0}(4 \times 2) & { }^{0}(2 \times 2)\end{array}\right]$.

Being, from (6),

$$
\operatorname{ker}(\mathbf{C})=\operatorname{im}\left[\begin{array}{c|c}
\mathbf{0}_{(\mathbf{1} \times 5)} & \mathbf{0}_{(1 \times 6)} \\
\mathbf{I}_{5} & \mathbf{0}_{(5 \times 6)} \\
\hline \mathbf{0}_{(2 \times 5)} & \mathbf{0}_{(2 \times 6)} \\
\mathbf{0}_{(6 \times 5)} & \mathbf{I}_{6}
\end{array}\right],
$$

it is an easy matter to show that $\operatorname{im}(\mathbf{J}) \subseteq \operatorname{ker}(\mathbf{C})$.

In [5] it is proven that the $\mathrm{im}(\mathbf{J})$ an is $(\mathbf{A}, \mathbf{B})$-controlled invariant, thus $\operatorname{im}(\mathbf{J}) \subseteq \max \mathcal{V}(\mathbf{A}, \mathrm{B}, \operatorname{ker}(\mathbf{C}))$.

Since $\operatorname{im}(\mathbf{D}) \subseteq \operatorname{im}(\mathbf{J})$, it follows that necessary and sufficient condition for disturbance localization holds,

$$
\mathrm{D} \subseteq \mathbf{J} \subseteq \mathrm{V}^{*}=\max \mathcal{V}(\mathrm{A}, \mathrm{B}, \operatorname{ker}(\mathrm{C}))
$$

As regards the stability requirement of the controlled couple $(\mathbf{A}+\mathbf{B F}, \mathbf{B})$, in [5] authors prove that there exists a stabilizing feedback matrix $\mathbf{F}$ which makes the controlled invariant im $(\mathbf{J})$ invariant with respect to $\mathbf{A}+\mathbf{B F}$.

\section{Case study}

A realistic simulation of a road vehicle with active suspensions is here reported to show an application of the disturbance decoupling theorem. The used parameters [4] of the vehicle geometry and dynamics are reported in Table 1 (cf. notation in Section 2).

The controller for the ride heights regulation is synthesised through a stabilizing state feedback matrix $F$, which localizes disturbances $d$ in the nullspace of the regulated output $\mathbf{y}=\left(\theta_{r}, \theta_{p}, z\right)$. Geometrically, the state feedback matrix $\mathbf{F}=-\mathbf{1 0}^{\mathbf{3}} \mathbf{G}$, where $\mathbf{G}$ is

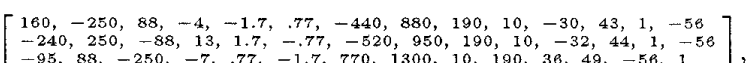
$\left[\begin{array}{ccc}-95,88,-250,-7, & 77,-17,770,1300,10,190,38,49,-56,3 \\ -170,-88,250,10,-77,1.7,700,1300,10,190,34,50,-56,1\end{array}\right]$,

satisfies these two properties,

\begin{tabular}{|c|c|}
\hline$l$ & $0.9 \mathrm{~m}$ \\
\hline$a$ & $2 \mathrm{~m}$ \\
\hline$M_{b}$ & $1500 \mathrm{~kg}$ \\
\hline$I_{r}$ & $360 \mathrm{kgm}^{2}$ \\
\hline$I_{p}$ & $2300 \mathrm{kgm}^{2}$ \\
\hline$M_{a 1}$ & $40 \mathrm{~kg}$ \\
\hline$M_{a 2}$ & $40 \mathrm{~kg}$ \\
\hline$I_{a 1}$ & $10.8 \mathrm{Kgm}$ \\
\hline$I_{a 2}$ & $10.8 \mathrm{Kgm}$ \\
\hline$K$ & $18 E 4 \mathrm{~N} / \mathrm{m}$ \\
\hline$\beta$ & $1 E 3 \mathrm{Ns} / \mathrm{m}$ \\
\hline$K_{t}$ & $1.96 \mathrm{E} 5 \mathrm{~N} / \mathrm{m}$ \\
\hline$\beta_{t}$ & $1.92 E 3 \mathrm{Ns} / \mathrm{m}$ \\
\hline
\end{tabular}

Table 1: Parameters of vehicle geometry and dynamics; spring and damping coefficients.

a) the (A, B)-controlled invariant $\mathbf{J}$ becomes $(\mathbf{A}+$ BF)-invariant;

b) the controlled invariant $\mathbf{J}$ is stabilized internally and externally.

The influence of the external disturbances, due to road surface irregularities, is simulated. Let us suppose that the vehicle has a constant speed of $60 \mathrm{~km} / \mathrm{h}$ and that the variation of the road surface profile occur every $16 m$ on the right side of the car $\left(d_{1} \neq 0 ; d_{3} \neq 0 ; d_{2}=\right.$ $d_{4}=0$ ). Assume that the front and rear wheels pass the same path, thus $d_{1}=d(t)$ and $d_{3}=d\left(t-T_{c}\right)$ where the delay $T_{c}$ is equal to $0.24 \mathrm{~s}$.

A ride of 10 seconds has been simulated, with and without the state feedback, under the action of a disturbance vector $\mathbf{d}(\mathbf{t})$ built as follows:

$$
d_{1}(t)=d(t) ; \quad d_{3}(t)=d\left(t-T_{c}\right) ; \quad d_{2}(t)=d_{4}(t)=0,
$$

where disturbance signal $d(t)$ is described, as a function of the time, in Figure 2.

Figure 2 reports the behaviour of the regulated outputs, roll, pitch angles and vehicle height. The plotted outputs are those relative to both system with and without feedback action F. As it is expected, variations of roll and pitch angles and of the vehicle height, due to disturbance $d$, disappear when the disturbance decoupling gain matrix $\mathbf{F}$ is fed back. Figure 3 illustrates the signals performed by active suspensions and commanded by the disturbance decoupling controller.

On the implementation side we cannot assume that the state vector is accessible, however it's not difficult to obtain a sensor system able to measure the suspensions heights along with their derivatives. As regards these latter variables, grouped in the sensed vector $\mathbf{y}_{s}=\mathbf{C}_{\mathbf{s}} \mathbf{x}$, it has been proved [5] that the lin- 

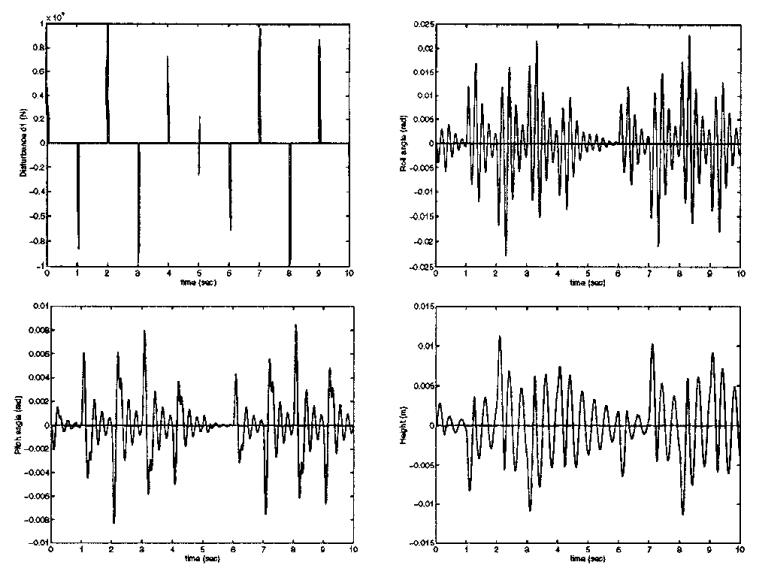

Figure 2: Disturbance $d(t)$ caused by the road surface variations (less than $0.05 m$ ). Roll, pitch angles and vehicle height for a ride of 10 seconds during which the disturbance $d_{1}$ and $d_{3}$ are exerted on the vehicle. Both outputs for systems with and without disturbance decoupling are reported. Signals identically zero refer to the vehicle with the state feedback gain $\mathbf{F}$.

earized dynamics (5) is observable from $\mathrm{y}_{s}$ and that the $(\mathbf{A}, \mathbf{B})$--controlled invariant im $(\mathbf{J})$ results to be an $\left(\mathbf{A}, \operatorname{ker}\left(\mathbf{C}_{\mathbf{m}}\right)\right)$-conditioned invariant subspace as well. It's worthwhile to mention that conditioned invariance of $\operatorname{im}(\mathbf{J})$ allows the designer to localize disturbances $d(t)$ by means of an output feedback gain instead of a state feedback one.

\section{Conclusions}

Localization of external disturbances in road vehicles with active suspensions was investigated. The problem of ride heights regulation, i.e. the regulation of the roll, pitch and vehicle height, was considered. The main result of the paper, Theorem 1 , states that there always exists a state feedback controller able to decouple external disturbances transmitted through suspensions.

The aim of this paper is to emphasize that such a decoupling property is a structural property of road vehicles with active suspensions. Moreover it's worthwhile to mention that only axes disturbances $\mathbf{d}$ enjoy the decoupling property. In fact it's an easy matter to verify that it's not possible to make the regulated outputs insensitive to those disturbances which are directly exerted on the sprung mass as, for instance, the lateral accelerations.

Finally, let us remark that the aim of this paper con-
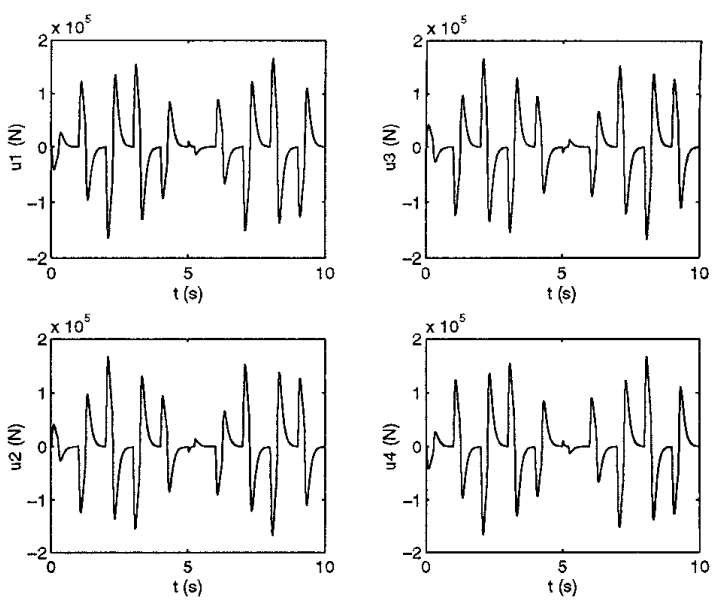

Figure 3: Active suspensions control outputs.

sists of enlightening some structural properties of vehicles with active suspensions more than synthesizing different algorithms taking into account various kinds of available actuators' dynamics.

\section{References}

[1] Basile G. and Marro G. "A state space approach to non-interacting controls," Ricerche di Automatica, vol. 1, n. 1, pp. 68-77, 1970.

[2] Basile G. and Marro G. Controlled and conditioned invariants in linear system theory, New Jersey, Prentice Hall, 1992.

[3] Hrovat D., "Optimal active suspension structures for quarter-car vehicle models," Automatica, vol. 26, no. 5 pp. $845-860,1990$.

[4] Peng H. and Tomizuka M., "Control of frontwheel-steering rubber tire vehicles," Report UCB ITS-PRR-90-5 of PATH programe, Institute of Transportation Studies, University of California at Berkley, USA, 1990.

[5] Prattichizzo D., Mercorelli P., Bicchi A., Vicino A., "A geometric approach to the control of vehicle with active suspensions," Internal Report CA-1-397, University of Siena, 1997.

[6] Unyelioglu K.A., Ozguner U. and Winkelman J., "A decomposition method for the design of active suspension controllers," in Proc. 13th 1996 IFAC World Congress, San Francisco USA, 1996.

[7] Wonham W.M. and Morse A.S., "Decoupling and pole assignment in linear multivariable systems: a geometric approach," SIAM J. Control, vol. 8, no. 1, pp. $1-18,1970$. 